\title{
A SUFFICIENT CONDITION FOR A MATRIC FUNCTION TO BE A PRIMARY MATRIC FUNCTION ${ }^{1}$
}

\author{
WALTER O. PORTMANN ${ }^{2}$
}

1. Introduction. A primary matric function is defined to be a matric function (that is, a mapping whose range and domain are sets of $n \times n$ matrices) arising from a scalar function of a complex variable. It has been shown [1] that primary matric functions are $H$-analytic. In this paper other necessary conditions for a primary matric function will be exhibited and it will then be shown that these conditions are also sufficient for a matric function to be a primary function.

We will first use a form of the definition of a primary function proposed by Frobenius and later use an equivalent form proposed by Giorgi [4]. Frobenius proposed that if the scalar function $f(z)$ is analytic at the eigenvalues of $Z$ in $\mathfrak{T}$ (the algebra of square matrices of order $n$ over the complex field) then $f(Z)$ shall be defined by

$$
f(Z)=\frac{1}{2 \pi i} \int_{C} \frac{f(\lambda)}{\lambda I-Z} d \lambda,
$$

where $C$ is a set of admissible closed paths enclosing each of the distinct eigenvalues of $Z$. That is, the components of $f(Z)$ are the integrals over $C$ of the corresponding components of the matrix $f(\lambda)(\lambda I-Z)^{-1} / 2 \pi i$.

We wish to exhibit sufficient conditions on a matric function $F(Z)$ such that there will exist a scalar function $g(z)$ for which $F(Z)=g(Z)$ where $g(Z)$ may be computed as in (1.1).

2. Necessary conditions. It has previously been shown in [1] that primary matric functions are $H$-analytic in $\mathscr{T}$, that is, the component functions of a primary function $g(Z)$ are analytic functions of the components $z_{i j}$ of $Z$, for $Z$ in an $M$-neighborhood of a matrix at which $g(Z)$ is defined.

If $g(z)$ is a scalar function defined at a matrix $X$, that is, $g(z)$ is analytic at the eigenvalues of $X$, and if $Y$ is such that for some nonsingular matrix $P, Y=P^{-1} X P$, then $g$ is defined at $Y$ and $g(Y)$ $=P^{-1} g(X) P$, as can be seen from (1.1).

If $Z$ is a matrix whose eigenvalues lie in the domain of analyticity of $g(z)$, then the $r, s$ component of $g(Z)$ is given by

Received by the editors January 3, 1959 and, in revised form, April 13, 1959.

1 This paper was prepared under the facilities granted by the Case Research Fund.

2 The author is now associated with Arizona State University. 


$$
g(Z)_{r s}=\frac{1}{2 \pi i} \int_{C} g(\lambda)(\lambda I-Z)_{r s}^{-1} d \lambda,
$$

where $(\lambda I-Z)_{r s}^{-1}$ is the $r, s$ component of $(\lambda I-Z)^{-1}$. For an upper triangular matrix $Z=\left(z_{i j}\right), z_{i j}=0$ for $i>j$, a simple computation shows that $(\lambda I-Z)_{r s}^{-1}$ and thus $g(Z)_{r s}$ depend only on the $z_{i j}$ for which $r \leqq i \leqq j \leqq s$ and is zero for $r>s$. In particular, $g(Z)_{r r}=g\left(r_{r r}\right)$ for $Z$ a diagonal (or upper triangular) matrix.

3. Sufficient conditions. We shall now show that these necessary conditions are also sufficient. For convenience the norm of a matrix $Z=\left(z_{i j}\right)$ shall be defined by norm $(Z)=\max _{i, j}\left|z_{i j}\right|$.

Theorem 3.1. Let $D$ be an open domain of $H$-analyticity of a matric function $F$ on $\mathfrak{T}$.

(i) Let $F$ be such that $X$ in $D$ and $Y=P^{-1} X P$ implies that $Y$ is in $D$ and $F(Y)=P^{-1} F(X) P$.

(ii) Let $F$ also be such that if $T=\left(t_{i j}\right)$, in $D$, is a diagonal matrix, then $F(T)_{r r}$ is a function of only $t_{r r}$, where $F(T)_{r r}$ is the $r, r$ component of $F(T)$, that is

$$
F(T)_{r r}=g_{r r}\left(t_{r r}\right) .
$$

Then there exists a scalar function $g(z)$ such that for all $Z$ in $D, g(Z)$ $=F(Z)$.

Proof. Let $C$ be a Jordan form for a matrix $Z$ at which $F$ is $H$ analytic, then $C$ is a direct sum $C_{p_{1}} \dot{+} \cdots+C_{p_{k}}$ of canonical blocks of the form

$$
C_{p_{i}}=\left(\begin{array}{cccccc}
\lambda_{i} & 1 & 0 & \cdots & 0 \\
& \lambda_{i} & 1 & & \cdot \\
& & \ddots & \cdot & \vdots \\
& & & \cdot & \lambda_{i} & \vdots \\
0 & & & & & \\
& & & & & \lambda_{i}
\end{array}\right)
$$

with $p_{i}$ rows and columns. (The $\lambda_{i}$ occurring in different $C_{p_{i}}$ need not be distinct.)

From (i) and Lemma 4.1 of [2] it follows that $F(C)$ commutes with all matrices that commute with the canonical matrix $C$. It is known that a matrix $F(C)$ satisfying this condition must be a direct $\operatorname{sum} P_{1}\left(C_{p_{1}}\right)+\cdots+P_{k}\left(C_{p_{k}}\right)$, where 


$$
P_{i}\left(C_{p_{i}}\right)=\left(\begin{array}{cccccc}
\alpha_{i_{1}} & \alpha_{i_{2}} & \alpha_{i_{3}} & \cdots & \alpha_{i_{p_{i}}} \\
& \alpha_{i_{1}} & \alpha_{i_{2}} & \cdots & \alpha_{i_{p_{i}-1}} \\
& & \alpha_{i_{1}} & \cdot & \cdot & \cdot \\
& & & \cdot & \cdot & \cdot \\
& & & & \cdot & \cdot \\
0 & & & & \alpha_{i_{1}} & \alpha_{i_{2}} \\
& & & & & \alpha_{i_{1}}
\end{array}\right)
$$

and $\alpha_{i_{m}}=\alpha_{j_{m}}$ for $\lambda_{i}=\lambda_{j}$ (see Turnbull and Aitken [7]).

Now, using a definition proposed by $\mathrm{G}$. Giorgi which is equivalent to (1.1) [4] for $g(Z)$ where $g(z)$ is a scalar function, it is seen that the theorem will be proven if there exists a scalar function $g(z)$ such that, for $C=P^{-1} Z P$, where $Z$ is any matrix at which $F$ is $H$-analytic,

$$
\alpha_{j_{m}}=g^{(m-1)}\left(\lambda_{j}\right) /(m-1) !
$$

or,

$$
F(C)_{r_{j} r_{j}+i}=g^{(i)}\left(\lambda_{j}\right) / i !, \quad j=1, \cdots, k, i=0, \cdots, p_{j}-1,
$$

where $F(C)_{r_{j} r_{j}+i}$ is the $r_{j}, r_{j}+i$ component of $F(C)$ (that is, $F_{r_{j} r_{j}+i}$ evaluated at the components of $C$ ) and $r_{j}=1+\sum_{t=1}^{j-1} p_{t}$ for $1<j \leqq k$, $r_{j}=1$ for $j=1$. (This choice of $r_{j}$ proves (3.2) for components in the first row of each triangular block $P_{j}\left(C_{p_{j}}\right)$ of $F(C)$ associated with $C_{p_{j}}$ of $C$, which is all that is necessary, since in any such block, the values on any super diagonal are all equal.)

We shall first exhibit a scalar function $g(z)$ which is determined by $F$ and then show that this function has the required property (3.3).

Let $Z$ be an arbitrary but fixed matrix such that $F$ is $H$-analytic in a neighborhood of $Z$; then $Z$ is similar to an upper triangular matrix $X=\left(x_{i j}\right)$ whose eigenvalues are the $x_{i i}$. By (i) $F$ is $H$-analytic in a neighborhood of $X$. Choose any matrix $Y=\left(y_{i j}\right)$ such that $y_{i j}=x_{i j}$ and $y_{i i} \neq y_{j j}$ for $i \neq j$, and $\left|y_{i i}-x_{i i}\right|<\epsilon$, where $\epsilon$ is sufficiently small such that $F$ is $H$-analytic at $Y$ (such an $\epsilon$ exists since $F$ is $H$-analytic in a neighborhood of $X) . Y$ is similar to a diagonal matrix $A=\operatorname{diag}\left(y_{k k}\right)$ with distinct eigenvalues $y_{i i}$ and by (i) $F$ is $H$-analytic at $A$. Now, $A$ is similar to a diagonal matrix $B$ obtained from $A$ by permuting, say $y_{i i}$ and $y_{j j}$, and by (i), this same permutation is performed on $F(A)$ in order to obtain $F(B)$. Thus by (ii), $g_{i i}\left(y_{j j}\right)=F(B)_{i i}=F(A)_{j j}$ $=g_{j j}\left(y_{j j}\right)$. Hence for any $j, g_{i j}(z)=g_{i i}(z)$ for $i=1, \cdots, n$ and $\left|z-x_{j j}\right|$ $<\epsilon$ and therefore, since the $F_{i \text { r }}$ are analytic, there exists a function $g(z)=g_{11}(z)\left(=g_{i i}(z), i=2, \cdots, n\right)$, analytic in the open circular domains $\left|z-x_{j j}\right|<\epsilon, j=1, \cdots, n$, where the $x_{j j}$ are the eigenvalues of $Z$. Thus, since $Z$ is an arbitrary matrix in $D$, there exists a function $g(z)$ which is analytic at the eigenvalues of all matrices in $D$. 
In order to show that $g(z)$ satisfies (3.3) we first note, from (3.1), that if $F$ is $H$-analytic in a neighborhood of a canonical matrix $C$, then $F(C)$ may be written

$$
F(C)=\sum_{i=1}^{k} \sum_{s=0}^{p_{i}-1} \sum_{t=0}^{p_{i}-s-1} F(C)_{r_{i} r_{i}+t} E_{r_{i}+s r_{i}+s+t}
$$

where $r_{i}=1+\sum_{t=1}^{i-1} p_{t}$ and $E_{p q}$ is the matrix with a 1 in the $p, q$ position and zeros elsewhere.

Now, for each $j, 1 \leqq j \leqq k$, let

$$
K_{p_{j}}=\left(\begin{array}{cccccc}
\lambda_{j} & 1 & 0 & \cdots & 0 \\
& \lambda_{j}+h_{j} & 1 & & & \cdot \\
& & \lambda_{j}+2 h_{j} & \cdot & \cdot & \cdot \\
& & & \ddots & \cdot & 1 \\
0 & & & & \lambda_{j}+\left(p_{j}-1\right) h_{j}
\end{array}\right)
$$

then for all $h_{j} \neq 0$ sufficiently small, $F$ is $H$-analytic at $K=K_{p_{1}}+\cdots$ $+K_{p_{k}}$ (since $F$ is $H$-analytic in a neighborhood of $C$ ).

Let $Q_{j}=\left(q(j)_{r s}\right), r, s=1, \cdots, p_{j}$, where $q(j)_{r s}=0$ for $r>s$ and $q(j)_{r s}=(-1)^{r+s} /(s-r) ! h_{j}^{s-r}$ for $r \leqq s$, then $Q_{j}^{-1}=\left(\tilde{q}(j)_{r s}\right)$ where $\tilde{q}(j)_{r s}=0$ for $r>s$ and $q(j)_{r s}=1 /(s-r) ! h_{j}^{s-r}$ for $r \leqq s$; also

$$
Q_{j} K_{p_{j}} Q_{j}^{-1}=D_{p_{j}}=\operatorname{diag}\left(\lambda_{j}+(i-1) h_{j}\right), \quad i=1, \cdots, p_{j} .
$$

Now, let $Q=Q_{1} \dot{+} \cdots+Q_{k}$, then $Q K Q^{-1}=\Lambda=D_{p_{1}} \dot{+} \cdots+D_{p_{k}}$, the canonical form of $K$. By (i), $F$ is $H$-analytic at $\Lambda$, and as in (3.4),

$$
F(\Lambda)=\sum_{i=1}^{n} F(\Lambda)_{i i} E_{i i}
$$

By (i), $F(K)=Q^{-1} F(\Lambda) Q$, therefore, for $0 \leqq i \leqq p_{j}-1, \quad F(K)_{r_{,} r_{j}+i}$ $=\sum_{s=0}^{i} q(j)_{r_{j} r_{j+8}} F(\Lambda)_{r_{j+s r_{j+8}}} q(j)_{r_{j}+s r_{j+i}}$. Thus, by the first part of this proof and the definitions of $q(j)_{r s}$ and $q(j)_{r s}$,

$$
\begin{aligned}
F(K)_{r_{j} r_{j}+i} & =\frac{1}{h_{j}^{i}} \sum_{s=0}^{i} \frac{(-1)^{s+i} g\left(\lambda_{j}+s h_{j}\right)}{s !(i-s) !} \\
& =\frac{1}{i ! h_{j}^{i}} \sum_{s=0}^{i}(-1)^{s+i}\left(\begin{array}{l}
i \\
s
\end{array}\right) g\left(\lambda_{j}+s h_{j}\right)=\frac{\Delta^{i} g\left(\lambda_{j}\right)}{i ! h_{j}^{i}} .
\end{aligned}
$$

Since $\lim _{h_{j} \rightarrow 0} \Delta^{i} g\left(\lambda_{j}\right) / h_{j}^{i}=g^{(i)}\left(\lambda_{j}\right)[6]$,

$$
\lim _{\Sigma_{j}\left|h_{i}\right| \rightarrow 0} K=C,
$$


and the $F_{r s}$ are analytic and therefore continuous in a neighborhood of the components of $C$, it follows that

$$
F(C)_{r_{j} r_{j}+i}=\lim _{h_{j} \rightarrow 0} F(K)_{r_{j} r_{j}+i}=g^{(i)}\left(\lambda_{j}\right) / i !
$$

Thus (3.3) is proven and hence Theorem 3.1.

It might here be noted that (i) alone is not sufficient for $F(Z)$ to be a primary matric function, as is shown by the function $F(Z)$ $=\sum_{i=1}^{n} F_{i i} E_{i i}$, where $F_{i i}=\sum_{k=1}^{n} z_{k k}=\operatorname{tr}(Z)$. The component functions $F_{i j}$ are analytic functions of the $z_{r s}$ of $Z$ and therefore $F$ is $H$ analytic; also, for $Y=P^{-1} Z P, F(Y)=P^{-1} F(Z) P$. However $F_{i i}$ is not a function of only $z_{i i}$ when $Z$ is a diagonal (or upper triangular) matrix which is necessary for a primary matric function.

It might be further noted, since $F(X)$ is diagonal when $X$ is diagonal, that if $X$ is restricted to the algebra $D$ of $n \times n$ diagonal matrices, then $F(X)$ is also a function on $\mathfrak{D}$. Ringleb [5] gave a necessary and sufficient condition for a function to be $H$-analytic in an algebra; namely, the (analytic) component functions must satisfy a certain set of linear homogeneous partial differential equations of the first order with constant coefficients which depend only on the structure of the algebra. For the algebra $D$, this necessary and sufficient condition for a function $F(T)=\sum_{i=1}^{n} F(T)_{i i} E_{i i}$ to be $H$-analytic in $D$ at a matrix $T=\operatorname{diag}\left(t_{j j}\right)$ is

$$
\frac{\partial F(T)_{i i}}{\partial t_{j j}}=0
$$

for $i \neq j$.

Thus hypothesis (ii) of Theorem 3.1 could be restated as follows: Let $F$ also be such that, when restricted to the algebra $D, F$ is $H$-analytic in $D$ at any diagonal matrix in $D$.

\section{REFERENCES}

1. W. O. Portmann, A derivative for Hausdorff-analytic functions, Proc. Amer. Math. Soc. vol. 10 (1959) pp. 101-105.

2. - Hausdorff-analytic functions of matrices, Proc. Amer. Math. Soc. vol. 11 (1960) pp. 97-101.

3. H. Richter, Über Matrixfunktionen, Math. Ann. vol. 122 (1950) pp. 16-34.

4. R. F. Rinehart, The equivalence of definitions of a matric function, Amer. Math. Monthly vol. 62 (1955) pp. 395-414.

5. F. Ringleb, Beitrage zur Funktionentheorie in hypercomplexen Systemen I, Rend. Circ. Mat. Palermo vol. 57 (1933) pp. 311-340.

6. D. W. Robinson, An application of the decomposition of a matrix into principal idempotents, Amer. Math. Monthly vol. 65 (1958) pp. 694-695.

7. H. W. Turnbull and A. C. Aitken, An introduction to the theory of canonical matrices, Blackie and Son Ltd., 1932.

Case Institute of Technology 